\title{
Avaliação da rede de monitoramento de uma bacia hidrográfica do Rio Grande do Sul, Brasil
}

\author{
Laís Fernandes de Moraesa, Osvaldo Cabongo ${ }^{\mathrm{b}}$ e Cristiano Poleto ${ }^{\mathrm{a}}$ \\ a Programa de Mestrado Profissional em Rede Nacional em Gestão e Regulação de Recursos Hídricos, Universidade Federal do Rio \\ Grande do Sul, Rio Grande do Sul, Porto Alegre, 91501-970, Brasil.*laisfmoraes@gmail.com \\ b Universidade do Vale do Rio dos Sinos, Rio Grande do Sul, São Leopoldo, 93022-750, Brasil.
}

Recebido: 23 fevereiro 2018 / Aceito: 20 maio 2018 / Publicado online: 28 maio 2018

\begin{abstract}
Resumo
O Rio dos Sinos é um dos rios mais poluídos do Brasil. Este artigo apresenta um levantamento de informações a respeito da rede de monitoramento hidrológico da Bacia Hidrográfica do Rio dos Sinos, estado do Rio Grande do Sul. Almejou identificar as diversas ações institucionais para avaliação da qualidade de água do rio. Foi realizada uma pesquisa documental por intermédio da qual se identificou os entes responsáveis pelo monitoramento hidrológico do Rio dos Sinos e seus afluentes. Constatou-se que há diversos órgãos responsáveis e que são variadas as frequências de amostragem de qualidade da água. A principal entidade responsável por esse monitoramento é a Fundação Estadual de Proteção Ambiental, que realiza a análise de parâmetros físicos, químicos e biológicos trimestralmente. Os titulares dos serviços de saneamento verificam em frequência horária, diária e mensal a água captada e os padrões dos efluentes lançados no rio. Outras entidades, estaduais e federais, realizam o monitoramento em estações fluviométricas e pluviométricas. Constatou-se, então, que o rio é altamente monitorado, porém não há integração dos dados. Os resultados de qualidade são disponibilizados com lapso temporal de três meses, e os resultados diários não são divulgados pelas companhias de saneamento ao público em geral.
\end{abstract}

Palavras-chave: Qualidade de água, gestão de recursos hídricos, sistemas de informação geográfica, Rio dos Sinos.

\section{Evaluation of the monitoring network of a river basin of Rio Grande do Sul, Brazil}

\begin{abstract}
The Sinos River is one of the most polluted rivers in Brazil. This article presents a survey of information about the hydrological monitoring network of Sinos River Basin, state of Rio Grande do Sul. It aimed to identify the institutional actions in progress to evaluate the water quality of the river. A documentary research was conducted through which the entities responsible for the hydrological monitoring of the Sinos River and its tributaries were identified. It was found that there are several responsible organisms and that the sampling frequencies of water quality are varied. The main entity responsible for this monitoring is the State Foundation for Environmental Protection, which performs the analysis of physical, chemical and biological parameters in quarterly frequency. The owners of the sanitation services verify the hourly, daily and monthly frequency of the adduced water and the effluent standards dumped in the river. Other entities, state and federal, perform the monitoring in fluviometric and rainfall stations. It was verified, then, that the river is highly monitored, but there isn't integration of the data. The quality results are available with a three-month time lapse and the daily results are not disclosed by the sanitation companies to the public.
\end{abstract}

Keywords: Water quality, water resources management, geographic information systems, Sinos River.

\section{Introdução}

A Bacia Hidrográfica do Rio dos Sinos está localizada na região nordeste do Rio Grande do Sul e inclui 32 municípios. A situação relativa ao conflito de uso da água, em termos de quantidade e qualidade, na bacia hidrográfica é preocupante. A garantia de uso para o abastecimento de mais de 1,3 milhões de habitantes e para a irrigação de lavouras, em contraponto aos usos que impactam a qualidade de suas águas, como os despejos de esgotos sanitários, diluição de efluentes industriais, agropecuários e drenagem urbana, afetam todo o componente ambiental da bacia do Sinos, contribuindo para tornar o manancial um dos mais poluídos do Brasil (Figueiredo, 2010).

A qualidade de água tem grande impacto na saúde pública, sendo essencial para o equilíbrio e o funcionamento dos ecossistemas. Carvalho (2014) considera fundamental que os componentes ambientais e suas concepções sistêmicas devam ser compreendidos de forma integrada. Dessa maneira, a água deve ser gerida considerando suas estreitas inter-relações com os outros componentes do meio (solos, vegetação, relevo) e com a ação antrópica, que altera as condições de funcionamento dos sistemas naturais, produzindo mudanças que podem afetar diretamente a qualidade e quantidade de água disponível (Carvalho, 2014). 
Tundisi et al. (2008) ressaltam que o conhecimento da qualidade das águas dos rios e do uso e da ocupação de suas bacias hidrográficas se faz necessário inclusive para traçar estratégias de planejamento e gestão, projetando cenários futuros, como o aumento da demanda de água e mudanças na paisagem decorrentes do desenvolvimento da região. Além disso, a gestão integrada de recursos hídricos deve ter por base uma abordagem participativa, envolvendo usuários, planejadores e formuladores de políticas em todos os níveis (Silva et al., 2017).

O planejamento da gestão de uma bacia hidrográfica deve propor uma visão que inclua as políticas públicas, as soluções tecnológicas e de educação ambiental a fim de promover o enfrentamento das problemáticas ambientais, a otimização de recursos e a garantia dos usos múltiplos da água. No entanto, as leis brasileiras reconhecem a qualidade da água tendo em vista apenas o seu grau de potabilidade e balneabilidade não considerando a integridade ecológica dos ecossistemas aquáticos que constituem as bacias hidrográficas (Callisto et al., 2012).

Tais apontamentos corroboram o fato de que a gestão dos recursos hídricos deve contemplar um conjunto de ações e medidas destinadas a regularizar o uso, o manejo e a proteção dos corpos d'água. Nesse sentido, é válido ressaltar que, para uma gestão eficiente dos recursos hídricos, é necessária a integração de projetos e atividades que visem avaliar e promover a recuperação e a preservação da qualidade e quantidade dos recursos hídricos (Américo-Pinheiro et al., 2016).

Diante do exposto, o objetivo deste artigo foi o de realizar o levantamento das informações sobre o monitoramento da qualidade da água, para fins de estabelecimento futuro de uma rede integrada de divulgação dos dados monitorados entre os entes atuantes na Bacia Hidrográfica do Rio dos Sinos.

\section{A Bacia Hidrográfica do Rio dos Sinos}

A Bacia Hidrográfica do Rio dos Sinos situa-se a nordeste do Estado do Rio Grande do Sul, entre as coordenadas geográficas de $29^{\circ} 20^{\prime}$ a $30^{\circ} 10^{\prime}$ de latitude Sul e $50^{\circ} 15^{\prime}$ a $51^{\circ} 20^{\prime}$ de longitude Oeste. Abrange as províncias geomorfológicas do Planalto Meridional e Depressão Central. Possui área de $3.696,53 \mathrm{~km}^{2}$, abrangendo municípios como Caraá, Campo Bom, Canoas, Gramado, Igrejinha, Novo Hamburgo, São Leopoldo, Sapucaia do Sul, Taquara e Três Coroas, com população total estimada em 1.350.147 habitantes (COMITESINOS, 2018). Além do Rio dos Sinos, os principais corpos de água da bacia hidrográfica são o Rio Rolante, o Rio da Ilha e o Rio Paranhana. O Rio dos Sinos nasce no município de Caraá e desemboca no delta do Rio Jacuí. (SEMA, 2017).

O Rio dos Sinos é a fonte de abastecimento de água para cerca de 1.300.000 habitantes, com uma densidade populacional de cerca de 360 pessoas por quilômetro quadrado. As áreas urbanas mais densamente povoadas, de acordo com estimativa populacional para o ano de 2017 (IBGE, 2017), incluem as cidades de Novo Hamburgo com 249.508 habitantes, Canoas com 343.853 habitantes e São Leopoldo com 230.914 habitantes, que estão localizadas na porção baixa da bacia. A região possui uma produção muito diversificada: calçados e couro, metais-mecânicos, alimentos, petroquímica, madeira e mobiliário, turismo e hospitalidade (Blume et al., 2010). A porção localizada na parte mais baixa da bacia está sob forte pressão antropogênica, pois concentra a maior densidade populacional, um importante polo industrial e PIB médio de $\mathrm{R} \$ 38$ mil reais per capta (IBGE, 2018).

$\mathrm{O}$ modelo econômico e produtivo e a maneira desorganizada como se deu a ocupação do solo e das áreas da bacia hidrográfica do Rio dos Sinos, tem levado à contaminação crescente dos corpos hídricos e à degradação da flora e fauna original. As consequências dos desequilíbrios causados pelo desenvolvimento das cidades e das atividades produtivas culminaram, por exemplo, com a mortandade de mais de 85 toneladas de peixes, no ano de 2006 (COMITESINOS, 2018).

\section{Monitoramento da qualidade de água na bacia hidrográfica do Rio dos Sinos}

A avaliação da qualidade da água da bacia hidrográfica do Rio dos Sinos, por longo período foi realizada pela Fundação Estadual de Proteção Ambiental - FEPAM. As séries de dados disponibilizadas pela entidade apresentavam representatividade temporal e distribuição espacial, desde a nascente até a foz e foram escolhidos segundo padrões de usos e ocupação do solo. A partir de 2005, a frequência de amostragem passou a ser bimestral e as coletas prosseguiram até outubro de 2013, quando foi interrompida (FEPAM, 2017).

O Comitê de Gerenciamento das Águas do Rio dos Sinos foi criado pelo Decreto Estadual n ${ }^{\circ} 32.774$, de 1988, e sempre apresentou forte mobilização social e ambiental. Segundo o Plano de Bacia Sinos (COMITESINOS, 2014), estão previstas ações de longo prazo, dentro do Programa de Monitoramento quali-quantitativo das águas do rio, como o aperfeiçoamento sobre as condições das águas superficiais da bacia, por meio da ampliação da rede de monitoramento fluviométrico e de qualidade e do acompanhamento das metas de enquadramento. Ainda entre as recomendações encontra-se a necessidade de formalização de intercâmbio de informações entre os órgãos gestores da bacia e as operadoras do sistema de abastecimento público presentes na bacia hidrográfica.

O Consórcio Público de Saneamento Básico da Bacia Hidrográfica do Rio dos Sinos foi criado em 2007, após grande mobilização decorrente do desastre ambiental em 2006.

A atuação do Consórcio Pró-Sinos (PROSINOS, 2017), que contempla 24 municípios consorciados, também está voltada para estudos de natureza técnica e social nas áreas de meio ambiente, saneamento e educação ambiental. Atualmente, está sendo retomado pelo Grupo Técnico de Projetos e Captação de Recursos o projeto Monitoramento Ambiental e Reforço das Estruturas de Fiscalização Ambiental da Bacia dos Sinos.

\section{Análise de dados}

As instituições, sua abrangência e dados quantitativos gerados sobre o monitoramento da Bacia Hidrográfica do Rio dos Sinos estão relacionados na Tabela 1.

A ANA (Agência Nacional de Águas) realiza o diagnóstico hidrometeorológico no país operando cerca de 4.540 estações 
de monitoramento. Tal monitoramento possibilita mensurar o volume de chuvas, a evaporação da água, o nível e a vazão dos rios, a quantidade de sedimentos e a qualidade das águas nas estações: pluviométricas, evaporimétricas, fluviométricas, sedimentométricas e de qualidade da água. A ANA disponibiliza as informações oriundas desse monitoramento no Sistema - Hidroweb e no Sistema Nacional de Informações sobre Recursos Hídricos (SNIRH, 2017).

Tabela 1. Resumo das fontes de informação pesquisadas e tipos de dados obtidos sobre a Bacia Hidrográfica do Rio dos Sinos

\begin{tabular}{|c|c|c|c|c|}
\hline Instituição & Tipos & Abrangência & Dados & Período \\
\hline ANA & $\begin{array}{c}\text { HIDROWEB / } \\
\text { Banco de dados } \\
\text { SNIRH }\end{array}$ & Rio dos Sinos & $\begin{array}{l}\text { Estações fluviométricas e } \\
\text { pluviométricas em operação }\end{array}$ & $2016-2017$ \\
\hline FEPAM & $\begin{array}{l}\text { Série histórica e } \\
\text { planilhas de } \\
\text { resultados } \\
\text { (trimestral) }\end{array}$ & Rio dos Sinos & $\begin{array}{l}\text { Parâmetros Físicos: condutividade, } \\
\text { temperatura, transparência e } \\
\text { turbidez. Químicos: cloretos, } \\
\text { cobre, DBO, DQO, fenóis totais, } \\
\text { orto-fosfato, manganês, nitrogênio } \\
\text { amoniacal, nitrogênio orgânico, } \\
\text { sólidos totais, surfactantes } \\
\text { aniônicos e zinco. Biológicos: } \\
\text { E.coli, fitoplâncton, clorofila A. }\end{array}$ & $\begin{array}{l}2010 \mathrm{a} \\
2013 \mathrm{e} \\
2016 \mathrm{a} \\
2017\end{array}$ \\
\hline COMUSA & $\begin{array}{l}\text { Planilhas horárias de } \\
\text { resultados }\end{array}$ & Captação Água Bruta & Condutividade; $\mathrm{pH}$ e temperatura & $2015-2017$ \\
\hline SEMAE & $\begin{array}{l}\text { Planilhas diárias de } \\
\text { resultados }\end{array}$ & Captação Água Bruta & $\begin{array}{l}\text { Alcalinidade, condutividade, cor, } \\
\text { carbono orgânico livre, matéria } \\
\text { orgânica, dureza, pH, oxigênio } \\
\text { dissolvido, temperatura, turbidez. }\end{array}$ & $2015-2017$ \\
\hline CORSAN & Banco de dados & $\begin{array}{c}\text { Captação Água Bruta } \\
\text { (Taquara, Campo Bom, } \\
\text { Esteio, Canoas) }\end{array}$ & Não foram disponibilizados & - \\
\hline SEMA/DRH-RS & $\begin{array}{l}\text { Mapa interativo - } \\
\text { Sala de Situação }\end{array}$ & $\begin{array}{c}\text { Rios dos Sinos, Rolante e } \\
\text { Paranhana }\end{array}$ & Precipitação, meteorologia & eventual \\
\hline COMITESINOS & $\begin{array}{l}\text { Site, documentos } \\
\text { técnicos }\end{array}$ & Bacia do Rio dos Sinos & $\begin{array}{l}\text { Caracterização da bacia (área, } \\
\text { municípios, projetos) }\end{array}$ & eventual \\
\hline CEMADEN & $\begin{array}{l}\text { Mapas interativos e } \\
\text { gráficos das estações } \\
\text { hidrológicas e } \\
\text { pluviômetros } \\
\text { automáticos }\end{array}$ & $\begin{array}{c}\text { Municípios monitorados: } \\
\text { Igrejinha, Novo Hamburgo, } \\
\text { Rolante, Sapucaia do Sul e } \\
\text { Três Coroas }\end{array}$ & Nível dos rios / pluviometria & eventual \\
\hline CPRM & $\begin{array}{l}\text { Relatórios de risco } \\
\text { geológico e estações } \\
\text { hidrometeorológica } \\
\text { em operação/ANA }\end{array}$ & $\begin{array}{l}\text { Municípios monitorados: } \\
\text { Igrejinha, Ivoti, Novo } \\
\text { Hamburgo, São Leopoldo, } \\
\text { Sapucaia do Sul, Taquara e } \\
\text { Três Coroas }\end{array}$ & $\begin{array}{c}\text { Nível dos rios / risco de enchentes } \\
\text { e movimentos de massa }\end{array}$ & 2016 \\
\hline
\end{tabular}

As estações de monitoramento da rede nacional estão estrategicamente localizadas nas várias bacias hidrográficas brasileiras e são operadas por entidades parceiras e de capacidade reconhecida, como a Companhia de Pesquisa de Recursos Minerais (CPRM), o Centro Nacional de Monitoramento e Alertas de Desastres Naturais, a Companhia Ambiental do Estado de São Paulo (CETESB) e a FEPAM$\mathrm{RS}$, as quais realizam o monitoramento por meio de contrato com a ANA. Atualmente, são mantidas na Bacia Hidrográfica do Atlântico Sul cerca de 344 estações fluviométricas; 466 estações pluviométricas; 51 estações de qualidade da água; 52 estações sedimentométricas e 95 estações telemétricas (ANA, 2017).

Para a padronização dos procedimentos de coleta, frequência e análise das informações foi lançada em 2013 a Rede Nacional de Monitoramento de Qualidade de Águas (RNQA) no âmbito do Programa Nacional da Qualidade de
Água (PNQA). Assim, buscou-se em articulação com as entidades operadoras das redes estaduais tornar os resultados confiáveis e sujeitos à comparação (ANA, 2016). No Rio Grande do Sul a principal entidade responsável pelo monitoramento da qualidade de água é a FEPAM.

Especialmente na bacia hidrográfica do Rio dos Sinos, foi articulada a Rede Integrada de Monitoramento do Rio dos Sinos - Comitê Sinos, que operou de 1990 a junho de 1996 (FEPAM, 2017). Posteriormente, em janeiro de 2000, teve início a Rede Integrada do Pró-Guaíba, contando com a participação da CORSAN e do Departamento Municipal de Água e Esgotos de Porto Alegre (DMAE).

Em 2005, a frequência de amostragem no Rio dos Sinos passou a ser bimestral. Para avaliação do Índice de Qualidade de Água (IQA) do Rio dos Sinos, foram realizadas coletas em 10 pontos de amostragem, e considerados os seguintes parâmetros: oxigênio dissolvido (OD), demanda bioquímica de 
oxigênio (DBO), coliformes termotolerantes e metais pesados, durante os anos de 1990 a 2011 (FEPAM, 2017).

A partir dos levantamentos realizados por esta pesquisa junto à FEPAM, constatou-se que até o ano de 2011 as coletas de amostras de água foram realizadas em um total de 17 pontos localizados ao longo dos $190 \mathrm{~km}$ do Rio dos Sinos. Eram analisados parâmetros físicos (condutividade, temperatura, transparência e turbidez), químicos (cloretos, cobre, DBO, DQO, fenóis totais, orto fosfato, manganês, nitrogênio amoniacal, nitrogênio orgânico, sólidos totais, surfactantes aniônicos e zinco) e biológicos (Escherichia coli) T. Escherich, 1885, pelo Departamento de Laboratório da FEPAM e os dados armazenados e interpretados pelo Departamento de Qualidade da FEPAM.

No ano de 2016, o estado do Rio Grande do Sul, por intermédio da Fundação Estadual de Proteção Ambiental aderiu ao programa Qualiágua - ANA, de acordo com as diretrizes estabelecidas na Resolução no 903/2013 (ANA, 2013) e retomou as análises dos pontos cadastrados na Bacia Hidrográfica do Rio dos Sinos, monitoramento este, que havia sido interrompido (FEPAM, 2017).

Os resultados enviados pela FEPAM ao sistema do Programa Qualiágua - ANA somente são divulgados no portal Hidroweb após serem submetidos à validação. O portal Hidroweb é um banco de dados que reúne todas as informações coletadas pela rede hidrometeorológica e disponibiliza dados sobre cotas, vazões, chuvas, evaporação, perfil do rio, qualidade da água e sedimentos (ANA, 2017). Os parâmetros de qualidade disponibilizados no portal são: turbidez, $\mathrm{pH}$, condutividade elétrica e oxigênio dissolvido.

Apesar do elevado nível técnico das instituições responsáveis pelo monitoramento hidrometeorológico, algumas lacunas podem ser observadas, pois a rede monitora padrões de qualidade de água em frequência trimestral. De acordo com Vespucci (2016), cabe aos operadores de estações fluviométricas atualizarem sistematicamente o banco de dados com os registros realizados in locu.

Para a população, esses dados ficam disponíveis no portal Hidroweb de responsabilidade da ANA, que possibilita a visualização e download das estações de interesse. Entretanto, as informações de qualidad, possuem um lapso temporal de três meses, ou seja, em muitos casos estão defasadas quanto à condição atual, inviabilizando o acompanhamento de importantes informações que retratam as características do rio monitorado (Vespucci, 2016).

As companhias de saneamento presentes na bacia hidrográfica (CORSAN, COMUSA E SEMAE) realizam diariamente a avaliação da qualidade de água do Rio dos Sinos, nos seus pontos de captação de água bruta. No início de 2017, foi necessário o estabelecimento de uma rede de comunicação emergencial entre os operadores das estações de tratamento de água, em virtude do evento hidrometeorológico extremo ocorrido nas cabeceiras dos afluentes do Rio dos Sinos (DRH, 2017). Na ocasião, os munícipios localizados na porção média e baixa da bacia ficaram desabastecidos devido aos altíssimos valores de turbidez da água do rio.

Logo, há a necessidade de maior frequência de disponibilização dos resultados de qualidade de água na bacia do Sinos. Com o uso de softwares gratuitos de sistemas de informação geográfica (SIG), é possível construir mapas temáticos e interativos (Lange et al., 2016), organizando dados e informações territoriais, como captação de água, lançamentos de efluentes, pontos de monitoramento ambiental e outros usos. Para fins de apresentação ou visualização, os arquivos shape, também podem ser exportados (arquivos kmz) para outras plataformas ou softwares voltados a SIG (UFPEL, 2017) e para o Google Earth.

Nesse contexto, as geotecnologias desempenham um papel essencial para as pesquisas que pretendem compreender e demonstrar a dinâmica espacial e temporal em projetos ambientais. De acordo com estudo realizado por Lange et al. (2016) na Bacia Hidrográfica do Rio Paraná, onde foram utilizados softwares gratuitos de geoprocessamento e SIG, a análise integrada permitiu a ação na gestão territorial da bacia.

O mapa da Figura 1 situa os locais de monitoramento da qualidade de água, realizadas pela FEPAM no Rio dos Sinos e as estações fluviométricas operadas pela CPRM. Os resultados dos parâmetros de qualidade destes pontos serviram de base para o enquadramento do rio, quando do processo de elaboração do Plano de Bacia (COMITESINOS, 2014).

A utilização de uma ferramenta de acesso aos dados de monitoramento teria como principal função facilitar o acesso e o acompanhamento dos resultados de qualidade da água do Rio dos Sinos, os quais são produzidos pelos diferentes entes e usuários da bacia hidrográfica. Ademais, a integração dos dados de monitoramento da qualidade pode vir de encontro aos anseios do Comitê Sinos (metas de médio e longo prazo) e também com os objetivos do Projeto de Monitoramento Integrado da Bacia do Consórcio Pró-Sinos.

Segundo Alvim e Ronca (2007) a gestão das águas deve buscar uma ampla articulação entre as diversas políticas setoriais e instituições que atuam na bacia e que estão em conflito. Ressalta-se que apenas a atuação do comitê não é suficiente para atingir a gestão integrada da bacia hidrográfica.

A quantidade de agentes envolvidos na bacia hidrográfica do Rio dos Sinos e a atuação dos integrantes do Sistema Nacional de Gerenciamento de Recursos Hídricos (SNGRH) podem favorecer uma gestão mais descentralizada, participativa e integrada do setor de recursos hídricos integrada no sentido de que todos os agentes envolvidos devem participar do processo de tomada de decisões (Junqueira et al., 2011). Contudo, para que de fato a descentralização seja efetiva, é necessário que as decisões tomadas pelos comitês de bacias sejam implementadas e que a inércia e a falta de enfrentamento dos problemas ambientais pelos agentes executores sejam modificadas, de modo promover a melhoria da qualidade dos recursos hídricos (Cerqueira et al., 2016).

O conhecimento das condições quali-quantitativas do corpo hídrico, pelo bom funcionamento das redes de monitoramento hidrológico e o cadastramento das demandas pelos sistemas de outorga, o poder público reúne condições de controle e de gestão da água. Para que a gestão possa ser efetiva, os órgãos públicos responsáveis devem estar organizados e equipados, tanto em relação à base de dados quanto em relação às estruturas de fiscalização e de monitoramento (Porto, 2008). Somente assim, pode-se assegurar que as condicionantes estabelecidas durante os procedimentos de licenciamento ambiental e de outorga estejam sendo respeitados. 


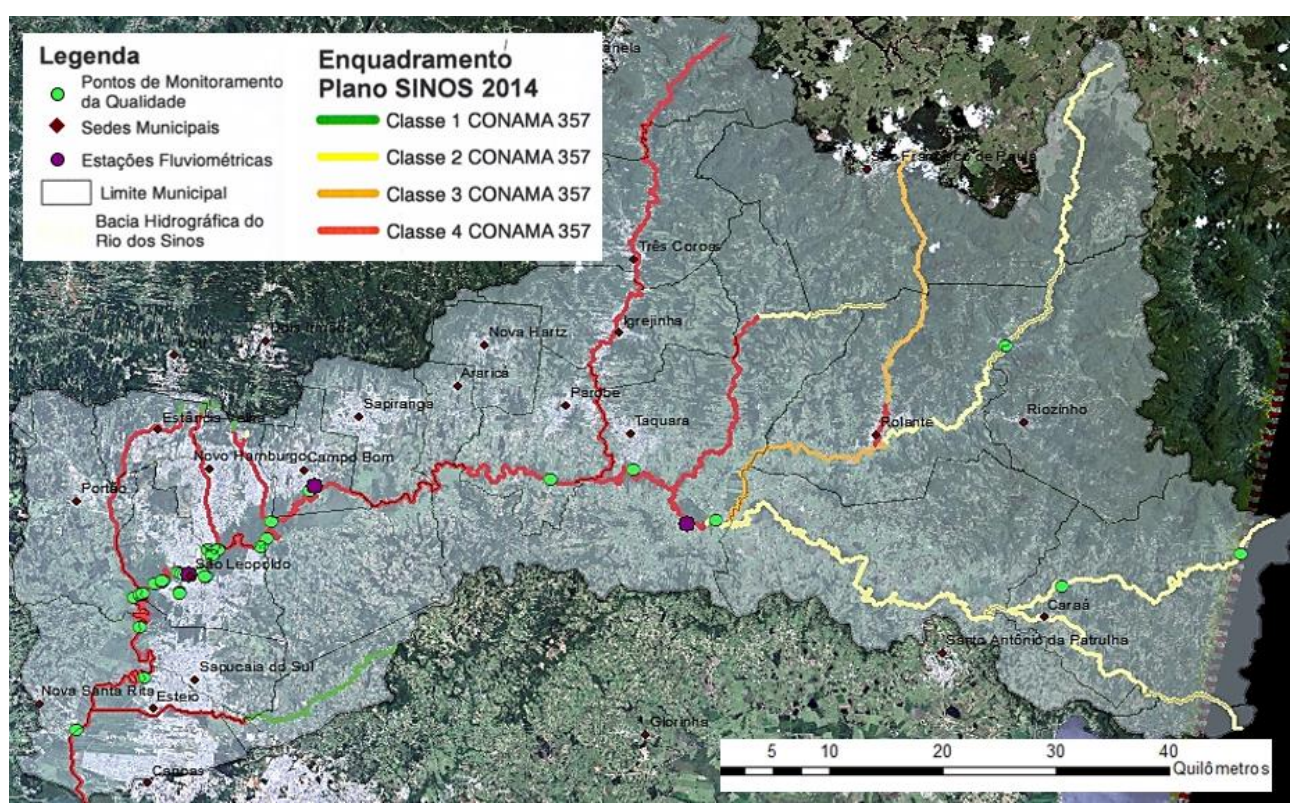

Figura 1: Bacia Hidrográfica do Rio dos Sinos. Elaborado segundo informações SIG do Plano de Bacia e com imagem do Google Earth.

\section{Conclusões}

Por meio dos dados revisados, esta pesquisa considerou que há melhora do monitoramento hidrometeorológico na bacia hidrográfica do Rio dos Sinos, pois existem estações pluviométricas e fluviométricas em operação, pelo CEMADEN, nos municípios de Rolante, Três Coroas, Igrejinha, Novo Hamburgo e Sapucaia do Sul e nas cidades de São Leopoldo, Campo Bom e Taquara, operados pela CPRM. Em 2017, outras estações automáticas de monitoramento foram instaladas pelo Departamento de Recursos Hídricos (SEMA-RS), para aumentar a área de abrangência e a prevenção a eventos hidrológicos extremos na referida bacia. Além da retomada em 2016 do monitoramento da qualidade realizado pela FEPAM em onze pontos ao longo do Rio dos Sinos.

Portanto, a implantação de uma ferramenta de integração dos dados da rede de monitoramento existente na Bacia Hidrográfica do Rio dos Sinos, ou seja, entre as instituições ANA, FEPAM, SEMA e companhias de saneamento, e a sua disponibilização por meio de ferramentas SIG devem contribuir para o gerenciamento e monitoramento ambiental na bacia, assim como, para a efetivação das metas e ações elencadas no Plano de Bacia. As informações apresentadas tornam-se importantes no sentido de permitir a participação e o controle social, a antecipação de ações de controle na fiscalização ambiental e no planejamento das metas estabelecidas para o enquadramento deste estratégico recurso hídrico.

\section{Referências}

Alvin A.T.B.; Ronca, J.L.C. 2007. Metodologia de avaliação qualitativa das ações dos Comitês de Bacias com ênfase na gestão integrada: o Comitê do Alto Tietê em São Paulo. Revista Engenharia Sanitária e Ambiental. 12(3): 325-334.

Américo-Pinheiro J.H.P.; Mirante M.H. P.; Benini S.M. 2016. Gestão e qualidade dos recursos hídricos: conceitos e experiências em bacias hidrográficas. Ed. ANAP, Tupã-SP, 95 p.

ANA, 2013. Resolução n ${ }^{\circ} 903$ de 22 de Julho de 2013. Cria a Rede Nacional de Monitoramento da Qualidade das Águas Superficiais e estabelece suas diretrizes, 10p. Acesso em: 22/07/2017

ANA, 2016. Conjuntura dos recursos hídricos: Informe/ Agência Nacional de Águas - Brasília: ANA. 95p. Acesso em: 22/07/2017

ANA, 2017. Portal da Qualidade das Águas. Acesso em: 24/07/2017.

ANA, 2017. Sistema de Informações Hidrológicas - HidroWeb. Acesso em: 04/08/2017

Blume, KK.; Macedo, J.C.; Meneguzzi, A.; Silva, L.B.; Quevedo, D.M.; Rodrigues, M.A.S. 2010. Water quality assessment of the Sinos River, Southern Brazil. Brazilian Journal Biology, 70(4): 1185-1193.

Callisto, M.; et al. 2012. Gestão eficiente de bacias hidrográficas no Brasil: dificuldades e perspectivas de soluções. Natureza \& Conservação, 10(1): $1-4$.

Carvalho, R.G. 2014. As bacias hidrográficas enquanto unidades de planejamento e zoneamento ambiental no Brasil. Caderno Prudentino de Geografia, Volume Especial (36): 26-43.

Cerqueira, L.S.; Fadul, E.; Vitória, F.T.; Morais J.L.M. 2016. Produção científica em gestão de recursos hídricos no Brasil no período de 2002 a 2011: uma análise da sua contribuição para o setor. Revista Gestão e Planejamento 17(2): 233-251.

COMITESINOS, 2014. Plano de Gerenciamento da bacia hidrográfica do Rio dos Sinos - META 3, Situação da situação Atual dos Recursos Hídricos. 148p. Acesso em: 25/08/2017

COMITESINOS, 2018. Bacia hidrográfica do Rio dos Sinos. Acesso em: $26 / 03 / 2018$

DRH. 2017. Diagnóstico Preliminar - Descritivo dos eventos ocorridos no dia 05 de janeiro de 2017 entre as regiões dos municípios de S. F. de Paula e Rolante - RS. Acesso em: 28/03/2017

FEPAM. 2017. Qualidade ambiental da Região Hidrográfica do Guaíba. Acesso em: 27/04/2017

Figueiredo, J.A.S.; Drumm, E.; Rodrigues, M.A.S.; Spilki, F.R. 2010. The Rio dos Sinos watershed: an economic and social space and its interface with environmental status. Brazilian Journal of Biology, 70(4): 1131-1136.

IBGE. 2018. Panorama Cidades. Acesso em: 18/03/2018.

Junqueira, M.A.D.R.; Saiani, C.C.S; Passador, C.S. 2011. Apontamentos sobre a lei brasileira das águas: a experiência do Estado de São Paulo. Revista de Gestão 18(2): 159-175.

Lange, M.V.; Andersen, S.M.; Pascoal, J.C. 2016. Territorial management for an appropriate technological choice: the case of $\mathrm{CIH}$ and CIBiogás projects in Parana 3 River Basin, Parana, Brazil. Renowable Energy and Power Quality. (14): 90-95

Porto, M.F.A.; Porto, R.L.L. 2008. Gestão de Bacias Hidrográficas. Estudos 
Avançados, São Paulo, 22(63): 43-60.

PROSINOS. 2017. Estatuto do Consórcio Público de Saneamento Básico da Bacia Hidrográfica do Rio dos Sinos - Pró-Sinos. Acesso em: 20/03/2017.

SEMA. 2017. Bacia Hidrográfica do Rio dos Sinos. Acesso em: 27/04/2017.

Silva, M.B.; Herreros, M.M.A.G.; Borges, F.Q. 2017. Gestão Integrada dos Recursos Hídricos como Política de Gerenciamento de Águas no Brasil. Revista de Administração da Universidade Federal de Santa Maria, 10.

SNIRH. 2017. Sistema de Informações de Recursos Hídricos. Acesso em $31 / 10 / 2017$

Tundisi, J.G.; Matsumura-Tundisi, T.; Pareschi, D.C.; Luzia, A.P.; Von Haeling, P.H.; Frollini, E.H. 2008. A bacia hidrográfica do Tietê/Jacaré: estudo de caso em pesquisa e gerenciamento. Estudos Avançados 22(63): 159-172.

UFPEL. 2017. Grupo de Pesquisa em Hidrologia e Modelagem Hidrológica em Bacias Hidrográficas. Software SYHDA. Acesso em: 06/10/2017

Vespucci, A.G.; Santos, J.G.R., Bayer, M. 2016. Estações fluviométricas do estado de Goiás: qualificação dos dados hidrológicos disponíveis na base HIDROWEB/ANA. Revista Ateliê Geográfico. 10(3): 89-108. 